\title{
Analyse spatio-temporelle de la fièvre typhoïde au niveau de la ville de Meknes (Maroc)
}

\section{Omar Mouhaddach}

\section{OpenEdition}

Journals

Édition électronique

URL : http://journals.openedition.org/rfst/495

DOI : $10.4000 /$ rfst.495

ISSN : 2492-3672

\section{Éditeur}

Espaces et SOciétés (UMR 6590)

Référence électronique

Omar Mouhaddach, «Analyse spatio-temporelle de la fièvre typhoïde au niveau de la ville de Meknes (Maroc) », Revue francophone sur la santé et les territoires [En ligne], Miscellanées, mis en ligne le 13 mai 2015, consulté le 06 avril 2021. URL : http://journals.openedition.org/rfst/495 ; DOI : https://doi.org/ $10.4000 /$ rfst. 495

Ce document a été généré automatiquement le 6 avril 2021.

\section{(i) 8 (2)}

La Revue francophone sur la santé et les territoires est mise à disposition selon les termes de la Licence Creative Commons Attribution - Pas d'Utilisation Commerciale - Partage dans les Mêmes Conditions 4.0 International. 


\title{
Analyse spatio-temporelle de la fièvre typhoïde au niveau de la ville de Meknes (Maroc)
}

\author{
Omar Mouhaddach
}

\section{NOTE DE L'ÉDITEUR}

Avec la collaboration de : Mohamed Ben-Daoud [contributeur], Université Moulay Ismail - Faculté des Sciences de Meknès (Maroc)Sophie Vanwambeke [contributeur], Université Catholique de Louvain - Louvain-la-Neuve (Belgique)Marie-Paule Kestemont [contributeur], Université Catholique de Louvain - Louvain-la-Neuve (Belgique) Samir El Jaafari [contributeur], Université Moulay Ismail - Faculté des Sciences de Meknès (Maroc).

\section{Introduction}

1 La dégradation de l'environnement peut être une des causes directes de plusieurs maladies telles que les cancers, les gastro-entérites, les affections respiratoires et cardiovasculaires (Chevalier et al. 2003). Selon l'Organisation Mondiale de la Santé, 24\% de la charge morbide et $23 \%$ de tous les décès sont imputables aux facteurs environnementaux. Parmi les enfants ( $0-14$ ans) la proportion des décès attribués à des causes environnementales s'élève à $36 \%$. Mais un grand nombre de ces facteurs environnementaux sont susceptibles d'être remédiés à l'aide de techniques, de programmes politiques et de mesures préventives (OMS, 2007).

2 Parmi ces maladies liées à l'environnement, la fièvre typhoïde constitue un problème majeur de santé publique, particulièrement dans les pays en voie de développement. Une estimation du fardeau de la fièvre typhoïde dans les pays à faible et moyen revenu en 2010 était de 11.9 millions cas et 129000 décès chaque année (Mogasale et al. 2014). 
3 La fièvre typhoïde est une maladie causée par une bactérie à réservoir strictement humain Salmonella typhi, sa transmission se fait principalement par voie oro-fécale notamment suite à la consommation d'aliments ou d'eau contaminés par des selles d'une personne infectée (Luby, 2014). Une série d'autres facteurs ont été signalés pour leur rôle dans la transmission de la maladie, tels que l'élimination inadéquate des eaux usées, le contact direct avec des porteurs asymptomatiques, le niveau de l'éducation, la proximité aux masses d'eau, l'hygiène personnelle, les conditions socio-économiques et le voyage dans les régions endémiques (Sur et al. 2007 ; Cheng et al. 2013 ; Dewan et al. 2013; Wang et al. 2013; Polonsky et al. 2014). En outre, les variables climatiques telles que la température, l'humidité et les précipitations ont un effet important sur la transmission et la répartition géographique de la fièvre typhoïde (Cheng et al. 2013; Dewan et al. 2013 ; Wang et al. 2013).

4 Au Maroc, le dernier bulletin épidémiologique des maladies à transmission hydrique ou alimentaire indique que le nombre des cas de la fièvre typhoïde a une tendance à la diminution depuis plusieurs années (DELM, 2008). Durant la période 2008-2010, les autorités sanitaires ont rapporté 1478 cas de la fièvre typhoïde dont 457 cas (soit 30\%) ont été déclarés par la région Meknès-Tafilalet (MS, 2009 ; MS, 2010 ; MS, 2011).

5 La ville de Meknès, chef lieu de la région Meknès-Tafilalet, avait contribué significativement à cette charge avec $65 \%$ des cas, malgré le fait qu'elle dispose d'une bonne infrastructure d'assainissement et d'eau potable. En effet, 95\% des ménages sont raccordés au réseau d'assainissement et environ $97 \%$ s'approvisionnent en eau potable depuis le réseau public.

6 Les déterminants de la fièvre typhoïde au niveau de la ville de Meknès sont très peu étudiés. Les professionnels de la santé suspectent en premier lieu l'extension des zones d'agriculture irriguées par des eaux usées au niveau urbain et périurbain de la ville. Néanmoins, les disparités socio-économiques, d'équipements de base et du niveau d'éducation peuvent jouer un rôle dans la répartition géographique de la maladie.

7 Par ailleurs, les techniques de statistique spatiale associées aux systèmes d'information géographiques (SIG) sont de plus en plus utilisées pour étudier la distribution spatiotemporelle des maladies en tenant compte des facteurs susceptibles de l'influencer. En effet, l'application de ces outils d'analyse spatiale en épidémiologie présente plusieurs avantages: une meilleure compréhension des processus de maladies; l'analyse des relations entre l'environnement et la présence de la maladie; la détection des agrégats de cas; la prédiction de la propagation de la maladie et l'évaluation des actions de contrôle et de prévention (Rezaeian et al. 2007 ; Tran et al. 2009 ; Carpenter 2011 ; Goria et al. 2011).

8 Des travaux de recherche ont déjà mobilisé ces outils pour identifier des agrégats spatiaux, des zones à risque et des facteurs associés à la fièvre typhoïde aux Etats-Unis (Hinman et al. 2006), l'Inde (Sur et al. 2007), le Népal (Baker et al. 2011), le Bengladesh (Corner et al. 2013 ; Dewan et al. 2013), la Chine (Cheng et al. 2013; Wang et al. 2013) et le Zimbabwé (Polonsky et al. 2014).

9 La présente étude vise à analyser la distribution spatio-temporelle de la fièvre typhoïde au niveau de la ville de Meknès durant la période 2008-2010, en tenant compte des facteurs susceptibles de l'influencer. Les hypothèses suivantes seront examinées : (i) l'incidence de la fièvre typhoïde n'est pas aléatoirement distribuée au niveau de notre zone d'étude ; (ii) le risque d'infection est élevé chez la population vivant à proximité 
des champs irrigués par des eaux usées; et (iii) les disparités locales d'équipements de base et du taux d'analphabétisme peuvent influencer la répartition géographique de la maladie.

Un état des lieux sur la pratique de la réutilisation des eaux usées en agriculture urbaine et périurbaine au niveau de la ville de Meknès, a été dressé pour mieux comprendre ce facteur environnemental.

\section{Matériels et méthode}

\section{Site d'étude : Ville de Meknès}

11 La ville de Meknès est située dans la partie septentrionale du Maroc, ses coordonnées géographiques sont : Longitude : $5^{\circ} 33^{\prime}$, Latitude : $33^{\circ} 52^{\prime}$ et Altitude : $530 \mathrm{~m}$.

12 Sur le plan administratif (Fig. 1), la ville regroupe quatre communes urbaines localisées sur le plateau du Saïs-Meknès, à savoir: Meknès, Al Machouar-Stinia, Ouislane et Toulal.

13 La population totale concernée par cette étude est estimée selon le Recensement Générale de la Population et de l'Habitat de 2004 à 563468 habitants et actualisée à 573589 habitants en 2010 (HCP, 2004).

$\mathrm{Du}$ point de vue social, certaines disparités socio-économiques existent entre les communes urbaines de la ville de Meknès avec des taux de pauvreté qui varient de 5.9\% (Al Machouar-Stinia) à 8.9\% (Toulal) (HCP, 2014). Les infrastructures publiques de soins couvrent l'ensemble de la ville, avec un réseau de 30 centres de santé de base répartis dans toutes les communes (AUM, 2013).

Figure 1 : Situation géographique de la ville de Meknès et son découpage administratif

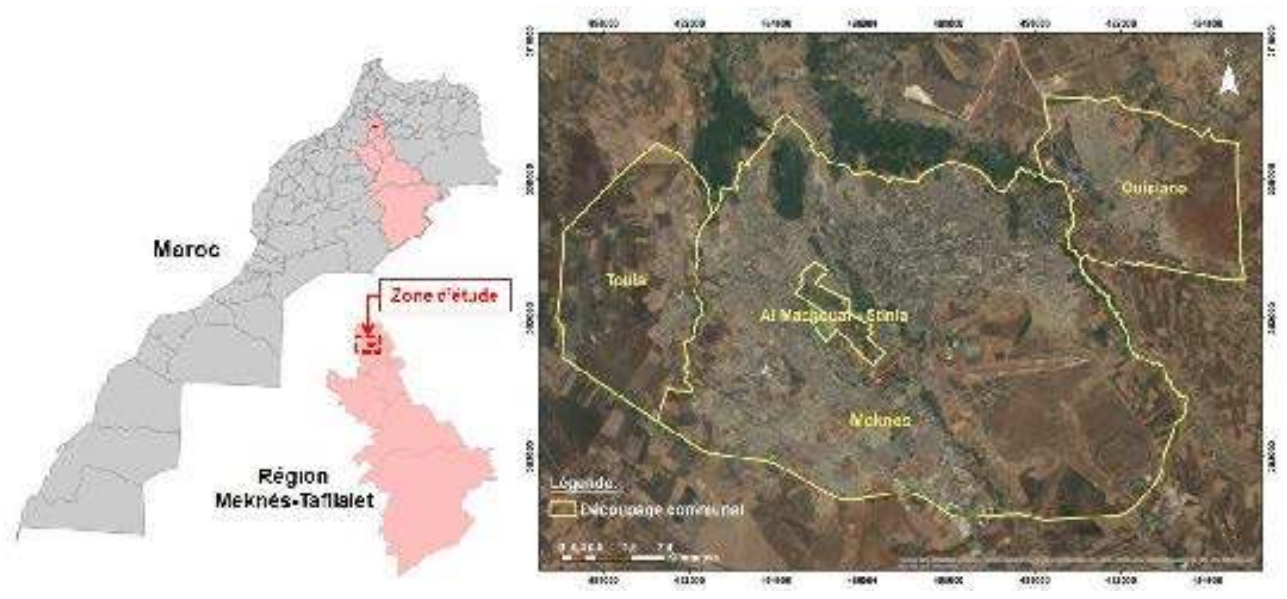

15 Doté de conditions naturelles (terres fertiles, climat tempéré) favorables et d'une infrastructure agroindustrielle, l'espace agricole de la ville de Meknès peut être considéré comme une locomotive du développement agricole de la région MeknèsTafilalet (DPA, 2007). Cependant, l'irrigation initialement basée sur l'eau des oueds et des sources, a été, suite à la raréfaction des ressources hydrauliques, renforcée par l'utilisation des eaux usées, ce qui présente actuellement des risques sanitaires pour l'homme et pour le bétail (Abdouh et al. 2004 ; Addouli et al. 2008). 


\section{Acquisition de données}

16 L'analyse rétrospective de la distribution spatiale et spatio-temporelle de la fièvre typhoïde au niveau de la ville de Meknès pour la période 2008-2010, s'est basée sur l'exploitation des données de différentes sources. Les données sanitaires portant sur les patients souffrant de la fièvre typhoïde ont été obtenues auprès de la Cellule Préfectorale d'Épidémiologie (CPE) de la préfecture de Meknès (Maroc). Les documents fournis comprennent les fiches journalières de déclaration autour des cas cliniques et confirmés de la fièvre typhoïde enregistrés au niveau des 2 hôpitaux régionaux et 30 centres de santé urbains de la ville de Meknès. Les informations exploitées par patient sont l'adresse du malade pour le géocodage et la date de déclaration pour l'analyse temporelle. Lorsque les informations sont mal renseignées sur les fiches, nous avons eu recours aux registres locaux des hôpitaux et des centres de santé pour les compléter.

17 Les données spatiales comprennent les polygones des zones urbanisées de la ville de Meknès à une échelle infra-communale et leur centroïde. En effet, nous avons réalisé un découpage infra-communal (Fig. 2) de la ville de Meknès, sur la base du découpage par district pour exploiter les données du Recensement Générale de la Population et de l'Habitat (RGHP) de 2004. Ce découpage a été conçu pour créer des zones homogènes en termes d'exposition au risque de consommation des aliments produits au niveau des champs irrigués par des eaux usées. Nous partons du constat que la majorité des citadins de la ville achètent généralement les légumes et les crudités du marché de leur quartier ou des quartiers voisins, bien qu'il existe une partie de la population (que nous estimons faible) qui se déplace plus loin pour s'approvisionner d'aliments.

Les centroïdes des zones urbanisées (Fig. 2) de chaque unité spatiale ont été générés pour calculer la distance d'éloignement des unités spatiales aux périmètres de la réutilisation des eaux usées en agriculture urbaine et périurbaine. 
Figure 2 : Découpage infra-communal de la ville de Meknès

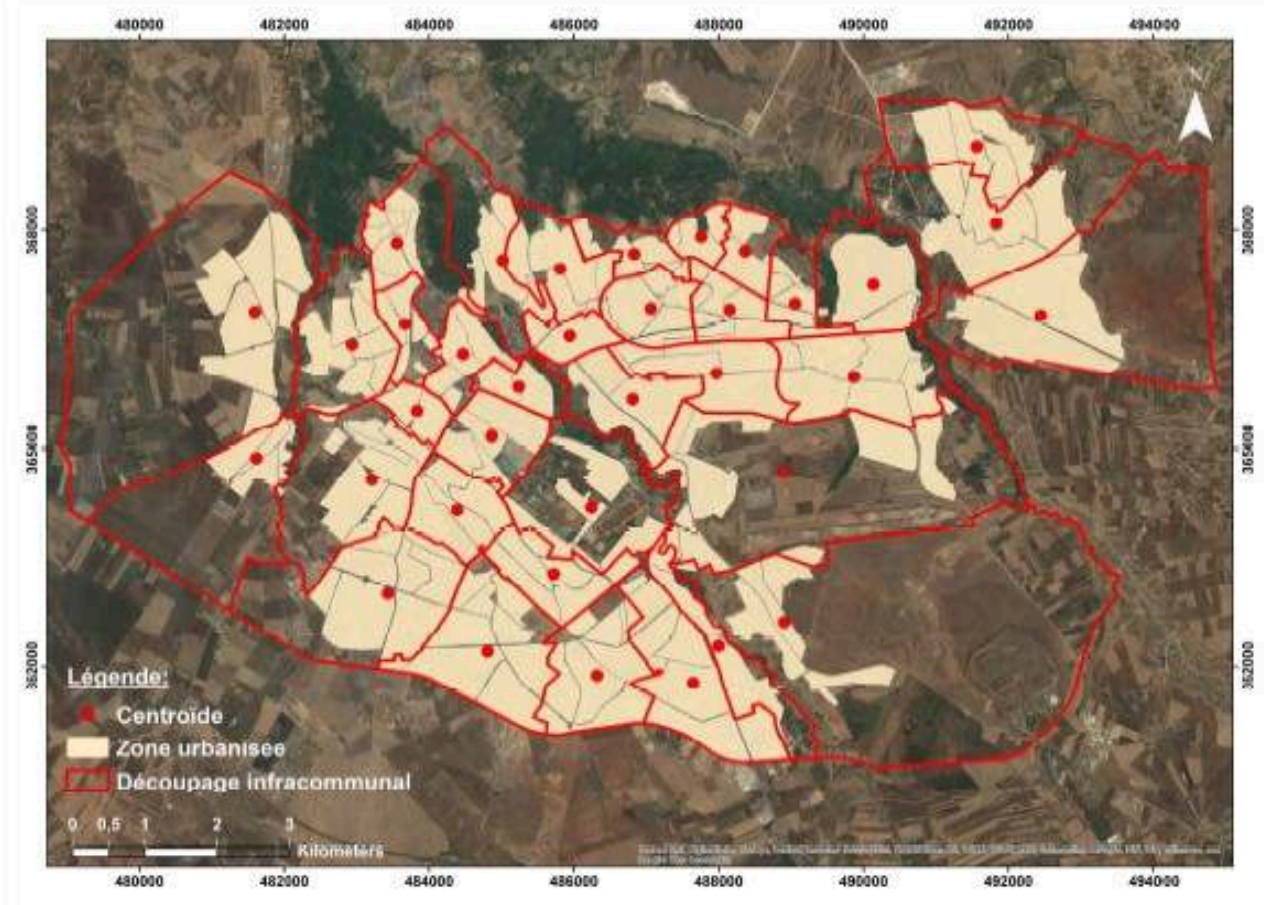

Les données de population à l'échelle infra-communale pour les années 2008, 2009 et 2010 ont été fournies par la direction régionale du Haut Commissariat au Plan (HCP) de Meknès.

20 Les données sur les facteurs suspectés d'être associés à la distribution la fièvre typhoïde au niveau de la ville de Meknès sont: le taux d'analphabétisme comme indicateur du niveau d'étude et le pourcentage de ménage ayant les équipements de base suivants : cuisine, toilette et bain moderne comme indicateur de la salubrité d'habitat. Ces données à l'échelle infra-communale sont issues du RGHP de 2004.

21 Les données environnementales sur la pratique de la réutilisation des eaux usées en agriculture urbaine sont quant à elles issues principalement d'un recensement des exploitations agricoles irriguées avec des eaux usées réalisé par la Régie Autonome de Distribution d'Eau et d'Electricité de Meknès et avec l'assistance de la Direction Provinciale de l'Agriculture de Meknès (RADEEM, 2003).

\section{Préparation des données}

Les données sanitaires et de population ont été utilisées pour calculer le taux d'incidence annuel de la fièvre typhoïde pour chaque unité spatiale selon l'équation suivante :

Taux annuel d'incidence $\left(\right.$ TAI $\left._{\mathrm{i}}\right)=$ Nombre de cas $\left(\mathrm{O}_{\mathrm{i}}\right) /$ Population $\left(\mathrm{P}_{\mathrm{i}}\right)$

Pour l'unité spatiale $\mathbf{i}$, le taux annuel d'incidence ( TAI ${ }_{i}$ ) pour une année donnée est égal au nombre de cas observés à cette unité $\left(\mathbf{O}_{\mathrm{i}}\right)$ divisé par la population attachée ( $\mathbf{P}$ i) à cette unité durant l'année en question.

25 L'exploitation des taux annuels d'incidences brutes dans les analyses spatiales peut conduire à des erreurs lorsque les nombres de cas sont faibles et quand les unités 
géographiques présentent des différences substantielles dans la taille des populations. Dans ces cas de figure, les taux annuels d'incidences brutes dans les différentes unités géographiques sont en effet d'un niveau de précision inégal et rendent de ce fait difficile la différenciation entre une fluctuation aléatoire et une vraie variation du niveau de risque entre les zones géographiques étudiées (Waller et Gotway, 2008 ; Goria et al. 2011).

Pour surmonter ce problème, un lissage des taux annuels d'incidences a été réalisé à l'aide de l'estimateur bayésien empirique du logiciel OpenGeoDa ( https:// geodacenter.asu.edu/ ). Cet estimateur utilise des principes bayésiens pour guider l'ajustement de l'estimation du taux brut en prenant en compte les informations dans le reste de l'échantillon (Anselin et al. 2006).

\section{Analyse et traitement des données}

\section{Analyse descriptive des données environnementales}

27 L'analyse descriptive des données portant sur la pratique de la réutilisation des eaux usées en agriculture urbaine au niveau de la ville de Meknès, a pour but de: (i) Déterminer les secteurs agricoles irrigués par des eaux usées brutes et mixtes (mélange des eaux conventionnelles avec les eaux usées); (ii) Comparer les superficies irriguées à partir des eaux usées brutes et mixtes entre les différents secteurs et (iii) Comparer l'occupation du sol par groupe de cultures entre les différents secteurs.

\section{Analyse globale d'autocorrélation spatiale}

28 L'autocorrélation spatiale représente les relations des régions adjacentes à l'égard du même attribut (le taux lissé d'incidence dans notre étude). De nombreux indices globaux d'autocorrélation spatiale ont été développés, dont les plus populaires sont: l'indice I de Moran, l'indice c de Geary et la statistique générale Gi (Getis et Ord, 1992 ; Pfeiffer et al. 2008). Ces indices permettent d'évaluer la tendance globale à l'agrégation de l'incidence d'une maladie dans une zone d'étude.

29 Considérant la ville de Meknès comme une seule entité, l'analyse globale d'autocorrélation spatiale à l'aide de l'indice I de Moran et la statistique générale Gi de Getis-Ord vise à examiner si la structure spatiale des taux d'incidences de la fièvre typhoïde est agrégée, dispersée ou aléatoire durant les années 2008, 2009 et 2010.

30 Les équations de l'indice global I de Moran (équation 1) et la statistique générale Gi de Getis-Ord (équation 2) s'expriment comme suit : 


$$
\begin{gathered}
\mathrm{I}=\frac{n}{\sum_{i=1}^{n} \sum_{j=1}^{n} w_{i j}} \times \frac{\sum_{i=1}^{n} \sum_{j=1}^{n} w_{i j}\left(x_{i}-\bar{x}\right)\left(x_{j}-\bar{x}\right)}{\sum_{i=1}^{n}\left(x_{i}-\bar{x}\right)^{2}} \\
G=\frac{\sum_{i=1}^{n} \sum_{j=1}^{n} w_{i, j} x_{i} x_{j}}{\sum_{i=1}^{n} \sum_{j=1}^{n} x_{i} x_{j}}, \forall j \neq i
\end{gathered}
$$
ij sont les pondérations reflétant les relations de proximité. Il faut noter que ces indices d'autocorrélation spatiale souvent signalés comme robuste posent le problème de la définition de la matrice de proximité car les résultats varient en fonction de la définition de cette matrice (Oliveau, 2010 ; Texier et al. 2011). d'indifférence", qui combine à la fois les caractéristiques des modèles "canal de distance » et "inverse de la distance ». Du fait qu'il offre l'avantage d'assurer une échelle d'analyse fixe qui favorise les unités voisines mais sans imposer une limite nette qui néglige l'effet des unités les plus éloignées. Pour appuyer ce choix, nous avons testé l'effet des différents modèles de relations spatiales sur les résultats des tests globaux.

\section{Analyse locale d'autocorrélation spatiale}

L'indice local de Moran (Anselin, 1995) et la statistique Gi* de Getis-Ord (Ord et Getis, 1995) ont été mobilisés pour mettre en évidence des associations locales des taux lissés d'incidence de la fièvre typhoïde à l'échelle infra-communale durant les années 2008, 2009 et 2010. En effet, ces indices décomposent les indices globaux de façon à identifier la contribution individuelle de chaque lieu, en mesurant successivement pour chacun la dépendance spatiale entre la valeur de la variable prise en ce lieu et l'ensemble de celles prises dans son voisinage (Oliveau, 2010). Ces indices locaux permettent d'identifier la localisation des clusters potentiels et tester si ces derniers sont significatifs.

L'indice local de Moran (équation 3) et la statistique Gi* de Getis-Ord (équation 4) ont été calculés comme suit : 


$$
\begin{gathered}
\mathrm{I}_{i}=\frac{n^{2}}{\sum_{i} \sum_{i} w_{i j}} \times \frac{\left(x_{i}-\bar{x}\right) \sum_{i} w_{i j}\left(x_{i}-\bar{x}\right)}{\sum_{i}\left(x_{j}-\bar{x}\right)^{2}} \\
\mathrm{G}_{i}(d)=\frac{\sum_{i=1}^{n} \sum_{i=1, i=1}^{n} w_{i j}(d) x_{i}}{\sum_{i=1}^{n} \sum_{j=1, j=1}^{n} x_{i}}
\end{gathered}
$$

\section{Analyse spatiale et spatio-temporelle par le scan spatial de Kulldorff}

Les méthodes de balayage cherchent à regrouper les différentes unités spatiales voisines en clusters potentiels à l'aide d'une fenêtre qui se déplace sur le plan géographique. Elles comparent l'incidence des cas à l'intérieur de cette fenêtre à celle de l'aire géographique restante (Texier et al. 2011).

Parmi ces méthodes, la statistique de scan spatial proposée par Martin Kulldroff (Kulldorff, 1997) est devenue la plus populaire. Cette méthode, basée sur un test du rapport de la vraisemblance, est très puissante et s'applique aussi bien sur des données groupées que ponctuelles (Kulldorff et al. 2006).

Dans le présent travail, le logiciel SatScan (http://www.satscan.org/) a été utilisé pour détecter, tester la significativité et identifier la localisation des zones à haut ou à bas risque de survenue de la fièvre typhoïde durant les années 2008, 2009 et 2010. L'analyse purement spatiale des agrégats a été réalisée en utilisant des fenêtres circulaires mobiles de tailles variables allant de 0 jusqu'à une valeur maximale spécifiée qui a permis l'incorporation de $25 \%$ de la population à risque. Pour la détection des agrégats spatio-temporels, le fenêtrage circulaire est transformé en cylindre dont la hauteur présente la dimension temporelle avec un maximum jusqu'à $50 \%$ de la période d'étude avec une précision de temps de 1 mois.

En supposant que les cas étaient distribués suivant la loi de Poisson, les agrégats spatiaux et spatio-temporels ont été évalués en comparant le risque d'incidence de la fièvre typhoïde à l'intérieur de la fenêtre avec le risque attendu si les cas de typhoïde étaient répartis au hasard dans l'espace et le temps. Le rapport de vraisemblance pour chaque agrégat a été calculé sur la base du nombre de cas à l'intérieur et à l'extérieur de la fenêtre. La fenêtre avec le rapport de vraisemblance maximale a été choisie comme l'agrégat le plus probable. L'importance d'agrégats spatiaux et spatio-temporels identifiés a été testée en utilisant le test du rapport de vraisemblance et les valeurs $p$ obtenues par 999 simulations de Monte Carlo, le seuil de signification a été fixé à 0.01 . 


\section{Analyse de la corrélation non paramétrique} mésuser l'association entre le taux d'incidence de la typhoïde et les facteurs susceptibles d'influencer sa distribution au niveau de la ville de Meknès durant la période 2008-2010.

En absence des données sur les indicateurs socio-économiques (comme le taux de pauvreté) à notre échelle d'étude, l'analyse a porté seulement sur le taux d'analphabétisme comme indicateur du niveau d'étude ainsi que sur le pourcentage de ménages ayant les équipements de base suivants: cuisine, toilette et bain moderne comme indicateur de la salubrité d'habitat. Concernant le facteur environnemental, nous avons supposé que les personnes vivant près des champs irrigués par des eaux usées avaient un risque plus élevé d'infection par la fièvre typhoïde que les personnes vivant plus loin. La distance entre le centroïde des zones urbanisées de chaque unité infra-communale (Fig. 2) et les périmètres de la réutilisation des eaux usées en agriculture, a été calculée pour construire l'indicateur environnemental.

\section{Résultats}

\section{Analyse descriptive des données sur la réutilisation des eaux usées en agriculture}

41 La ville de Meknès possède l'un des plus importants périmètres de réutilisation des eaux usées brutes et eaux mixtes (mélange des eaux conventionnelles avec les eaux usées), avec une superficie d'environ 2245 ha. Les zones de réutilisation des eaux usées se répartissent sur les vallées des trois cours d'eau suivants (Fig. 3): Bouishak, Boufekrane et Ouislane (RADEEM, 2003 ; Addouli et al. 2008). Avec des nettes différences en termes de débit dont le plus important est l'oued Boufekrane aussi bien en raison de son débit (236 l/s en étiage) que de son déploiement au cœur de la ville. Les deux autres oueds Ouislane à l'est et Bouishak à l'ouest de la ville drainent des débits d'étiage moins importants, respectivement de l'ordre de $131 \mathrm{l} / \mathrm{s}$ et $40 \mathrm{l} / \mathrm{s}$ (ABHS, 2008). 
Figure 3 : Espace agricole urbain et périurbain de la ville de Meknès

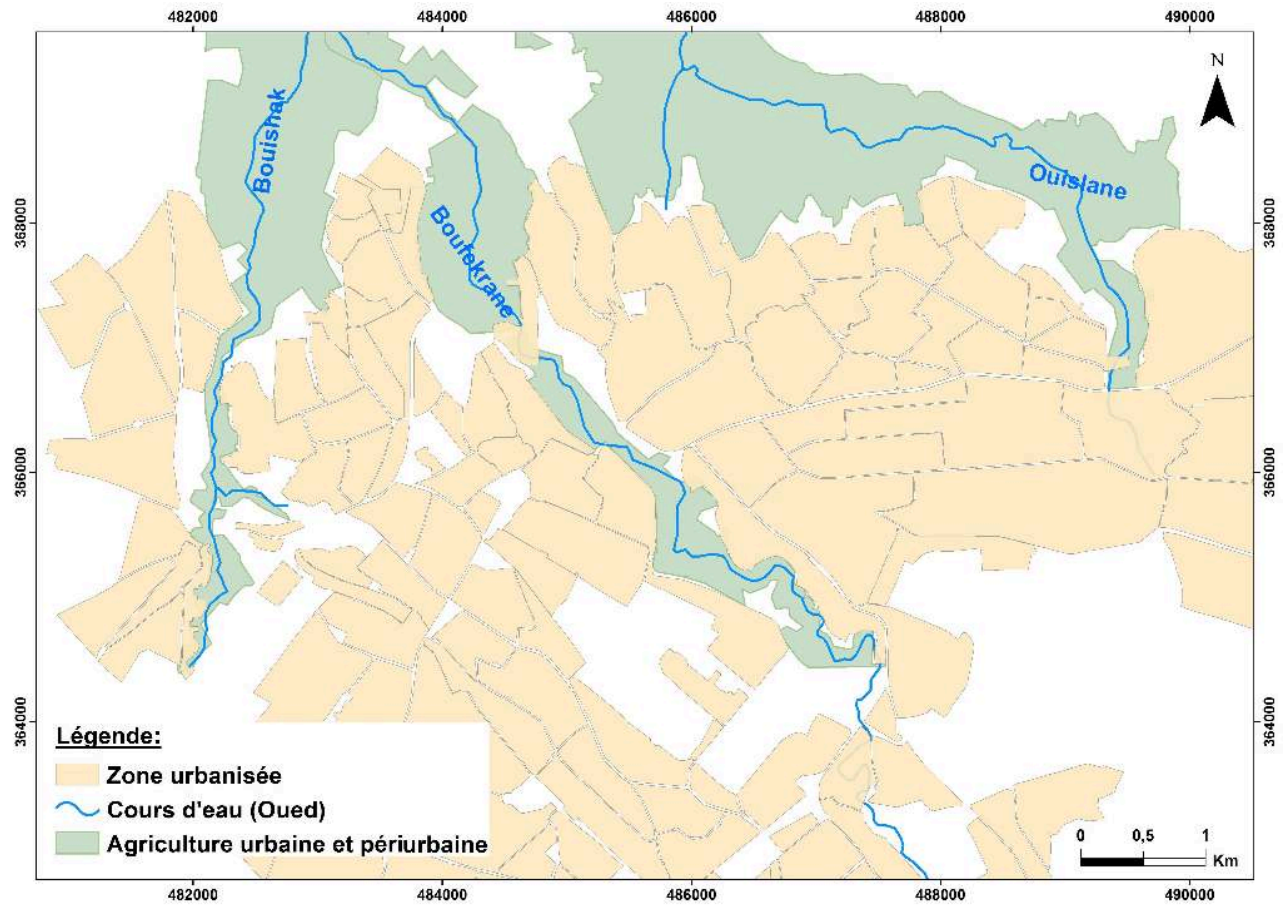

Les superficies agricoles irriguées par des eaux usées et des eaux mixtes au niveau des trois secteurs atteignent $95,4 \%$ de l'ensemble des superficies recensées, avec une dominance des surfaces irriguées exclusivement par des eaux usées brutes $(77,5 \%)$. L'analyse des terrains irrigués par ces eaux à l'intérieur de chaque secteur (Fig. 4) a montré que le secteur Bouishak est le plus touché par la pratique d'irrigation par les eaux usées brutes avec un pourcentage de $93 \%$, suivi par le secteur Ouislane $(71 \%)$ et finalement le secteur Boufekrane (69\%).

Figure 4 : Pourcentage des terrains irrigués avec les différents types d'eau

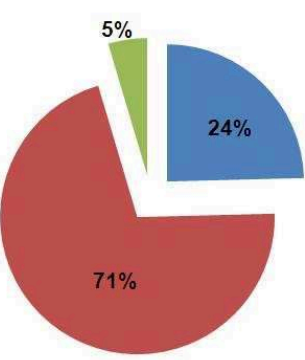

$\underline{\text { Ouislane }}$

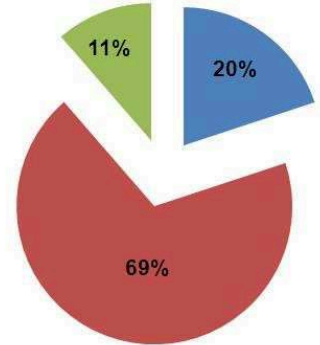

Boufekrane

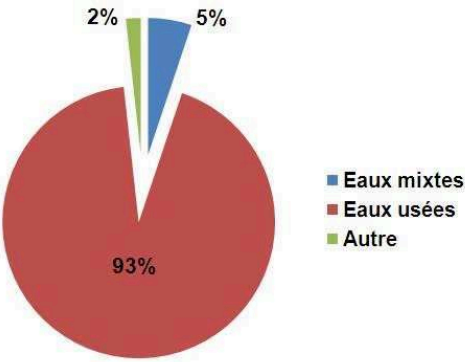

Bouisshak

Ces possibilités d'irrigation dans les trois secteurs ont abouti à la diminution de la jachère à $1,2 \%$ de la surface agricole utile, et une diversification des cultures. L'analyse de la répartition des cultures et plantations (plein champ et intercalaires) par groupes de cultures (Fig. 5) ressort que: (i) Les cultures maraîchères totalisent $53 \%$ de la surface agricole utile, et sont développées dans les secteurs Ouislane et Bouishak; (ii) Les cultures fourragères occupent environ $14 \%$ de la surface agricole utile; (iii) Les céréales et légumineuses restent peu développées, elles sont importantes dans le secteur Ouislane ; (iv) Les plantations occupent environ $49 \%$ de la surface agricole utile et sont bien développées dans les trois secteurs : Bouishak, Boufekrane et Ouislane. 
Figure 5 : Occupation du sol par groupe de cultures

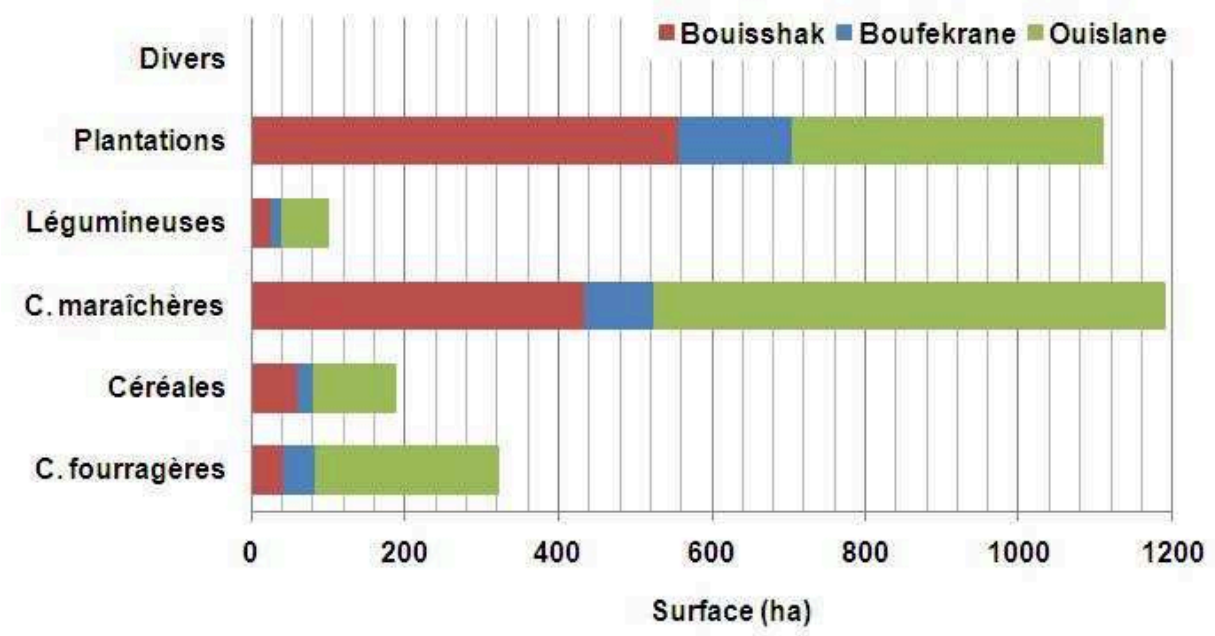

Ces productions diversifiées alimentent directement et régulièrement et en quantité importante les différents marchés informels qui foisonnent dans la ville sans passer forcement par le marché de gros de fruits et légumes.

\section{Analyse d'autocorrélation spatiale de la fièvre typhoïde}

Durant la période 2008-2010, la ville de Meknès avait enregistré 299 cas, soit environ $20 \%$ des cas de fièvre typhoïde rapportés à l'échelle nationale pour la dite période.

Les résultats de l'analyse globale d'autocorrélation spatiale des estimations des taux annuels d'incidence de la fièvre typhoïde par l'indice I de Moran et la statistique générale Gi de Getis-Ord sont représentés dans le tableau 1.

Ces résultats montrent que nous pouvons rejeter l'hypothèse nulle qui correspond à une structure totalement aléatoire des incidences de la maladie pour les années 2008 et 2010 avec un niveau de signification de 0.05 et 0.01 respectivement. Pour l'année 2009, la distribution des incidences observées présente une structure aléatoire. Les valeurs positives des z-scores générés par la statistique générale Gi indiquent que l'agrégation se fait au niveau des valeurs élevées d'incidence.

Donc, l'analyse globale d'auto-corrélation spatiale a révélé la tendance des incidences élevées de la fièvre typhoïde à l'agrégation durant les années 2008 et 2010, contre leur dispersion durant l'année 2009 qui avait enregistré le nombre de cas le plus faible ( 57 cas) par rapport aux années 2008 ( 96 cas) et 2010 ( 146 cas). Il faut noter que le changement du modèle de relations spatiales n'a pas affecté les résultats des indices globaux. Pour cela, nous avons opté pour le modèle «zone d'indifférence » pour les analyses locales d'autocorrélation spatiale. 
Tableau 1 : Résultats des indices globaux d'autocorrélation spatiale

\begin{tabular}{lccccc} 
& \multicolumn{2}{c}{$\begin{array}{c}\text { Global I de } \\
\text { Année }\end{array}$} & \multicolumn{2}{c}{$\begin{array}{c}\text { Statistique } \\
\text { Moran }\end{array}$} & \multicolumn{2}{c}{ Générale Gi } & \multirow{2}{*}{ Profil } \\
\cline { 2 - 5 } & $\begin{array}{c}\text { Indice } \\
\text { I }\end{array}$ & $\begin{array}{c}\text { Z- } \\
\text { score }\end{array}$ & $\begin{array}{c}\text { Indice } \\
\text { G }\end{array}$ & $\begin{array}{c}\text { Z- } \\
\text { score }\end{array}$ & \\
\hline 2008 & 0,16 & $2,25^{*}$ & 0,25 & $2,96^{*}$ & Agrégé \\
\hline 2009 & 0,09 & 1,14 & 0,17 & 1,09 & Dispersé \\
\hline 2010 & 0,35 & $4,80^{* *}$ & 0,35 & $5,07^{* *}$ & Agrégé \\
\hline *Niveau de signification est de 0.05 & & \\
\hline **Niveau de signification est de 0.01 & & \\
\hline
\end{tabular}

La figure 6 regroupe les résultats de l'analyse locale d'autocorrélation spatiale par l'indice local de Moran (LISA) et la statistique Gi* de Getis-Ord. L'indice local de Moran (Fig. 6a.c.e) a permis de ressortir des agrégats High-High qui correspondent à des régions avec une incidence élevée entourée par des voisins avec des incidences élevées de la maladie. Par ailleurs, la statistique Gi* (Fig. 6.b.d.f) a détecté des hots spots (zones à incidence élevée de la fièvre typhoïde) durant les 3 années d'études, contre un seul cold spot (zone à incidence faible) durant l'année 2008.

Plus précisément, pour l'année 2008 la statistique Gi* a révélé un hot spot formé de 4 secteurs au nord-ouest de la ville et correspond à un agrégat High-High détecté par l'indice local de Moran et un cold spot formé de 3 secteurs au sud de la ville (Fig. 6a.b). L'analyse des taux annuels d'incidence pour l'année 2009 a détecté un hot spot formé de 5 secteurs dont le plus significatif est à la limite nord-ouest de la ville. L'indice local de Moran a quant à lui révélé un cluster avec seulement 2 secteurs du dit hot spot et situé au centre de la ville à la rive ouest de la zone agricole Boufekrane (Fig. 6c.d). Pour l'année 2010, un hot spot de 7 secteurs a été détecté au nord-ouest de la ville, dont 3 secteurs forment un cluster High-High à proximité des secteurs agricoles Boufekrane et Ouislane (Fig. 6e.f). 
Figure 6 : Résultats de l'analyse locale d'autocorrélation spatiale des taux d'incidence de la fièvre typhoïde par l'indice local de Moran et la statistique $\mathrm{Gi}^{\star}$ de Getis-Ord

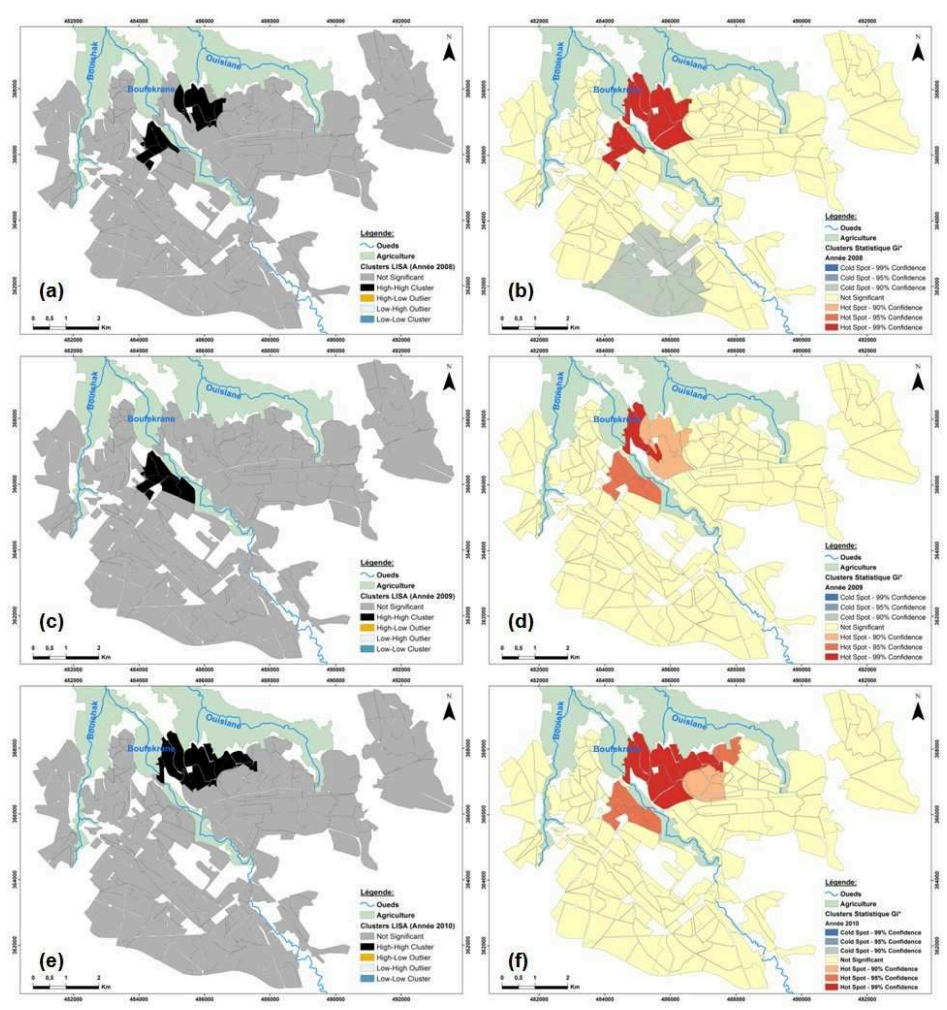

51 Nous remarquons que les deux techniques ont produit le même profil spatial avec des zones à haut risque d'infection par la fièvre typhoïde situées au nord-ouest de la ville, pas loin des zones agricoles irriguées par des eaux usées durant les années 2008, 2009 et 2010 .

\section{Analyse spatiale et spatio-temporelle par le scan spatial de Kulldorff}

Les cartes de la figure 7 représentent la localisation des agrégats spatiaux et spatiotemporels obtenus par la méthode du scan spatial, dont les secteurs en couleur rouge et bleu correspondent respectivement aux secteurs les plus touchés par la fièvre typhoïde de la ville et ceux qui sont épargnés de ce risque.

53 La détection des agrégats spatiaux par le test de scan spatial de Kulldorff a mis en évidence l'existence des clusters à haut et à bas risque, hautement significatifs $(p<0,01)$, avec des risques relatifs ${ }^{1}$ (RR) qui varient de 0.05 à 5.68 : (i) Pour l'année 2008 (Fig. 7a) le test a détecté un seul cluster à haut risque (H1) au nord-ouest qui présente un risque relatif de 5.62 et deux clusters à bas risque (L1) au sud et (L2) au nord-est avec des risques relatifs de 0.07 et 0.05 respectivement; (ii) Pour l'année 2009 (Fig. 7b) le test a mis en évidence deux clusters, dont l'un (H1) à haut risque au nord-ouest (RR=3.55), alors que l'autre (L1) à bas risque au nord-est $(\mathrm{RR}=0,17)$; (iii) Pour l'année 2010 (Fig. 7c) le test a détecté trois clusters, dont un est à haut risque $\mathrm{H} 1$ au nord-ouest avec un risque relatif de 5.68, et deux à bas risque $L 1(R R=0.12)$ au sud-est et $L 2(R R=0.19)$ au nord-est.

L'analyse spatio-temporelle de l'incidence de la fièvre typhoïde durant les années 2008 , 2009 et 2010, a permis de détecter des clusters à haut risque avec des risques relatifs 
allant de 7.09 à 11.95 : (i) Pour l'année 2008, l'analyse a mis en évidence deux clusters à haut risque au nord-ouest de la ville, H1 constitué de 7 secteurs avec un risque relatif de 9.00 durant la période Mai-Septembre 2008 et $\mathrm{H} 2(\mathrm{RR}=11.3)$ durant les mois Juillet et Août (Fig. 7d); (ii) Un cluster spatio-temporel à haut risque H1 (RR=07.09) formé de 7 secteurs et situé au nord-ouest de la ville a été détecté durant la période MaiOctobre 2009 (Fig. 7e); Pour l'année 2010, l'analyse a détecté un cluster à haut risque H1 $(\mathrm{RR}=11,95)$, formé de 8 secteurs et situé au nord-ouest de la ville durant la période MaiOctobre 2010 (Fig. 7f).

Figure 7 : Résultats de l'analyse spatiale et spatio-temporelle de la fièvre typhoïde par le scan spatial de Kulldorff

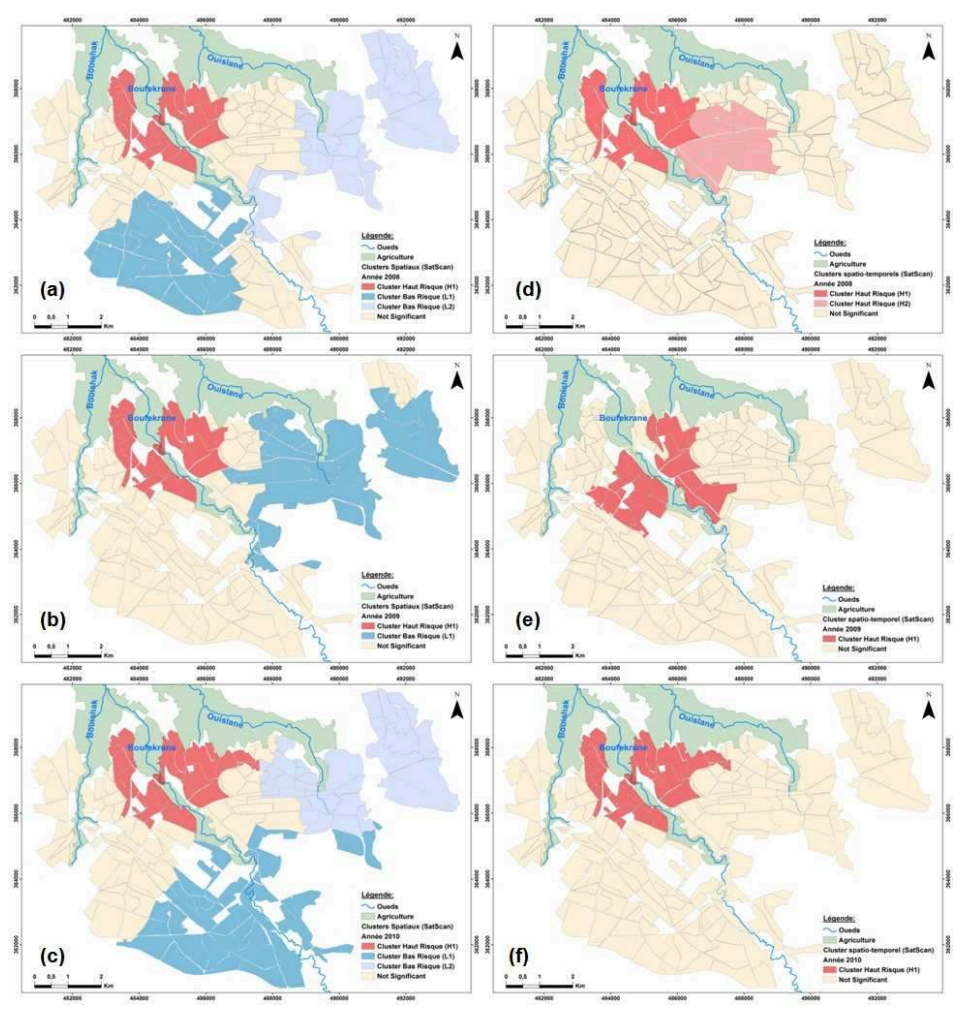

L'analyse des agrégats spatiaux et spatio-temporels durant la période 2008-2010, montre que les zones à bas risque d'infection par la fièvre typhoïde sont concentrées au niveau de la partie sud-est de la ville, loin des champs irrigués par des eaux usées. Alors que les zones à haut risque s'agrègent au nord-ouest et surtout durant la période MaiOctobre de chaque année.

\section{Analyse de la corrélation non paramétrique}

Le tableau 2 regroupe les coefficients de corrélation non paramétrique qui mesurent l'association entre le taux d'incidence de la fièvre typhoïde durant la période 2008-2010 et les facteurs associés à sa distribution au niveau de la ville de Meknès. Ces résultats montrent une association négative hautement significative $(\mathrm{p}<0.01)$ entre l'incidence de la fièvre typhoïde et la proximité aux trois secteurs agricoles irrigués par des eaux usées. 
57 L'analyse de la même association en prenant les secteurs agricoles deux à deux a révélé des associations négatives, dont la plus forte est la proximité aux secteurs Boufekrane et Bouishak.

Aucune association statistique n'a été trouvée avec le taux d'analphabétisme et le pourcentage des équipements de base : cuisine, toilette et bain moderne.

Tableau 2 : Résultats des coefficients de corrélation non paramétrique entre l'incidence de la fièvre typhoïde et les facteurs associés

\begin{tabular}{|c|c|c|}
\hline \multirow{2}{*}{$\begin{array}{l}\text { Facteurs associés à } \\
\text { la fièvre typhoïde }\end{array}$} & \multicolumn{2}{|c|}{$\begin{array}{c}\text { Coefficients de } \\
\text { corrélation non } \\
\text { paramétrique }\end{array}$} \\
\hline & $\begin{array}{c}\text { rho de } \\
\text { Spearman }\end{array}$ & $\begin{array}{l}\text { tau-b de } \\
\text { Kendall }\end{array}$ \\
\hline $\begin{array}{l}\text { Proximité aux secteurs } \\
\text { agricoles: Boufekrane } \\
\text { et Ouislane }\end{array}$ & $-0,428^{*}$ & $-0,298^{* *}$ \\
\hline $\begin{array}{l}\text { Proximité aux secteurs } \\
\text { agricoles: Boufekrane } \\
\text { et Bouishak }\end{array}$ & $-0,674^{* *}$ & $-0,467^{* *}$ \\
\hline $\begin{array}{l}\text { Proximité aux secteurs } \\
\text { agricoles: Ouislane et } \\
\text { Bouishak }\end{array}$ & $-0,353^{*}$ & $-0,269^{*}$ \\
\hline $\begin{array}{l}\text { Proximité aux trois } \\
\text { secteurs agricoles de } \\
\text { la ville }\end{array}$ & $-0,551^{* *}$ & $-0,390^{* *}$ \\
\hline $\begin{array}{l}\text { Taux d'analphabétisme } \\
\text { Pourcentage de }\end{array}$ & $-0,019$ & $-0,004$ \\
\hline $\begin{array}{l}\text { Ménages disposant de } \\
\text { cuisine } \\
\text { Pourcentage de }\end{array}$ & $-0,198$ & $-0,125$ \\
\hline $\begin{array}{l}\text { Ménages disposant de } \\
\text { toilette }\end{array}$ & $-0,048$ & $-0,043$ \\
\hline $\begin{array}{l}\text { Pourcentage de } \\
\text { Ménages disposant de } \\
\text { bain moderne }\end{array}$ & $-0,171$ & $-0,115$ \\
\hline $\begin{array}{l}{ }^{*} \text { La corrélation est signi } \\
\text { ** La corrélation est sign }\end{array}$ & $\begin{array}{l}\text { ative au nivea } \\
\text { cative au nive }\end{array}$ & $\begin{array}{l}0.05 \\
0.01\end{array}$ \\
\hline
\end{tabular}

\section{Discussion}

59 A notre connaissance, la présente étude est le premier travail au Maroc qui utilise les techniques de statistique spatiale associées aux SIG pour analyser la distribution spatiale et spatio-temporelle de la fièvre typhoïde à une échelle fine (infracommunale). Cette étude d'épidémiologie spatiale de la fièvre typhoïde au niveau de la ville de Meknès constitue une contribution à la compréhension de la distribution de la maladie au sein de la ville et à l'identification des facteurs de risque associés, en vue d'appuyer les efforts des autorités locales à la lutte et au contrôle de ce type de maladie à transmission hydrique ou alimentaire.

Les indices globaux d'autocorrélation spatiale ont permis de révéler la tendance des incidences élevées de la fièvre typhoïde à l'agrégation durant les années 2008 et 2010. Mais les indices locaux ont produit le même profil spatial des taux annuels d'incidence de la fièvre typhoïde durant les trois années d'étude. En effet, les régions de la ville à haut risque d'infection par la fièvre typhoïde sont situées au nord-ouest de la ville à la proximité des périmètres de la réutilisation des eaux usées en agriculture, notamment aux alentours des oueds Bouishak et Ouislane. 
61 Ces deux secteurs sont fortement touchés par la pratique de la réutilisation des eaux usées en irrigation et produisent des quantités importantes de crudités qui sont commercialisées localement au niveau de la ville. Ce constat sur la commercialisation des produits maraichères irrigués par des eaux usées au niveau des marchés locaux a été rapporté par des travaux d'enquêtes auprès des agriculteurs urbains de la ville de Meknès (Grégoire 2012 ; Rhaidour 2013).

62 La consommation des aliments irrigués par des eaux usées pose des risques sanitaires puisqu'ils peuvent être le vecteur des pathogènes associés aux excrétas, dont la bactérie responsable de la fièvre typhoïde la Salmonella typhi (Bos et al. 2011; OMS, 2012).

63 Pour confirmer le dit profil spatial de la distribution de la fièvre typhoïde, la méthode de balayage par le scan spatial du Kulldorff a été utilisée pour détecter des agrégats spatiaux et spatio-temporels. Cette technique a été mobilisée pour surmonter les limites des techniques d'autocorrélation spatiale, à savoir la sensibilité au choix du modèle de relation spatiale entre les voisins et le fait d'utiliser des taux lissés d'incidence générés par un estimateur bayésien empirique (Gaudart et al. 2007 ; Oliveau, 2010).

64 Le scan spatial de kulldorff a révélé la présence d'un gradient spatial de la distribution de la fièvre typhoïde par rapport aux champs irrigués par les eaux usées, dont les clusters à haut risque d'infection s'agrègent principalement au nord-ouest de la ville, et les clusters à bas risque au sud-est. Ce type de profil spatial de la fièvre typhoïde a été rapporté au niveau du district de Hongta (Chine) par Wang et al. (2013) en confirmant la diminution des cas de la fièvre typhoïde en s'éloignant des marchés qui commercialisent les produits irrigués par des eaux usées et des canaux d'irrigation pollués.

65 La prise en compte de la dimension temporelle dans l'analyse de scan spatial, a montré la récurrence de l'agrégation des zones à haut risque au nord-ouest durant la période Mai-Octobre. Cette période coïncide avec la hausse de la température et la raréfaction des ressources en eau ce qui induit un recours intensif à la réutilisation des eaux usées en agriculture.

66 En effet, le profil climatique de la ville de Meknès, montre un déficit pluviométrique annuel supérieur à $988 \mathrm{~mm}$, avec des déficits mensuels se situent entre $39 \mathrm{~mm}$ et $237 \mathrm{~mm}$ et se produisent 8 mois sur 12. Les plus forts déficits sont observés pendants les mois les plus productifs (de Mars à Septembre), ce qui pousse les agriculteurs à la réutilisation des eaux usées en irrigation durant cette période où les débits des cours d'eau sont faibles (Zitane, 2009 ; Ouattar, 2010).

67 En plus, plusieurs travaux de recherche (Aboulkacem et al. 2007; Ben-Daoud et al. 2011; Larif et al. 2013), sur la qualité des eaux superficielles au niveau de la ville de Meknès, ont rapporté une forte contamination bactériologique d'origine fécale des eaux des trois oueds, et particulièrement en aval de l'agglomération où les eaux sont utilisées à des fins agricoles.

68 Selon Charron et al. (2008), la sécheresse intensifie la demande en eau à des moments où le niveau des sources d'approvisionnement en eau est déjà considérablement abaissé et où celles-ci sont vulnérables. L'eau devient concentrée en agents pathogènes et en contaminants chimiques, ce qui a des conséquences pour les mesures d'hygiène étant donné les restrictions imposées relatives à l'utilisation de l'eau. 
De nombreuses études ont rapporté l'effet de la température sur la distribution temporelle des cas de la fièvre typhoïde avec une augmentation notable durant les périodes chaudes (Cheng et al. 2013 ; Dewan et al. 2013 ; Corner et al. 2013)

Une association négative hautement significative a été trouvée entre l'incidence de la fièvre typhoïde et la proximité aux secteurs agricoles irrigués par des eaux usées, alors qu'aucune association statistique n'a été trouvée avec le taux d'analphabétisme et le pourcentage des équipements de base : cuisine, toilette et bain moderne. Cela appuie l'hypothèse sur l'augmentation du risque d'infection par la fièvre typhoïde chez les populations vivant à proximité des champs irrigués par des eaux usées. Du fait que le facteur environnemental influence la répartition de la maladie plus que les facteurs niveau d'étude et salubrité d'habitat.

71 Au terme de cette discussion, il faut souligner certaines limites de notre étude :

72 Les données sanitaires sur les cas de la fièvre typhoïde sous-estiment la réalité pour plusieurs raisons. Parmi celles-ci, on mentionnera le fait que les individus malades ne consultent pas nécessairement un médecin, que certains cas sont asymptomatiques et qu'il existe une sous-notification des cas des hôpitaux privés;

73 Les données exploitées sur la pratique de la réutilisation des eaux usées en agriculture urbaine remontent à l'année 2003, ce qui nécessite leur actualisation pour tenir en compte de l'effet de l'urbanisation et des variations climatiques durant ces dernières années.

74 L'étude des facteurs associés à la fièvre typhoïde s'est limitée aux indicateurs du niveau d'étude et de salubrité d'habitat, en raison de la difficulté de mobiliser des données sur les facteurs socio-économiques à l'échelle infra-communale.

\section{Conclusion}

L'application des techniques de statistique spatiale associées aux SIG a permis de déterminer les zones de population à haut risque d'infection par la fièvre typhoïde au niveau de la ville de Meknès. En effet, ces zones sont situées à proximité des champs irrigués par les eaux usées. Les distinctions locales du taux d'analphabétisme et du niveau d'équipements de base ont été examinées comme facteurs d'influence probable de la répartition géographique de la maladie.

Les techniques appliquées constituent une étape importante dans la mise en œuvre des programmes locaux spécifiques à la prévention et contrôle de la fièvre typhoïde au niveau de la ville de Meknès, en permettant une économie de temps et d'efforts aux autorités compétentes. 


\section{BIBLIOGRAPHIE}

Abdouh M., El Atrouz A., Mechkouri A., 2004, Profil environnemental de Meknès, Agendas 21 locaux pour la promotion de l'environnement et du développement durable en milieu urbain, Rabat : Ministère de l'Aménagement du Territoire, de l'Eau et de l'Environnement.

Aboulkacem A., Chahlaoui A., Soulaymani A., Rhazi-filali, Benali D., 2007, Etude comparative de la qualité bactériologique des eaux des oueds Boufekrane et Ouislane à la traversée de la ville de Meknès (Maroc), REMISE, 1:1, p : 10-22,

Addouli J., Chahlaoui A., Chafi A., Berrahou A., 2008,Suivi et analyse du risque lié à l'utilisation des eaux usées en agriculture dans la région de Meknès au Maroc, Revue Sud Sciences \& Technologies, $\mathrm{N}^{\circ} 16$, p. 29-35.

Agence du Bassin Hydraulique de Sebou (ABHS), 2008, Monographie des ressources en eau de Meknès, Fès : Agence du Bassin Hydraulique de Sebou.

Agence Urbaine de Meknès (AUM), 2013, Etude du SDAU du grand Meknès et plan d'aménagement de l'agglomération de Meknès, Mission I : Diagnostic et Orientations, Meknès : Agence Urbaine de Meknès.

Anselin L, Lozano L, Koschinsky J, 2006, Rate transformations and smoothing. In Spatial Analysis Laboratory Department of Geography. University of Illinois: Urbana-Champaign

Anselin L., 1995, Local Indicators of Spatial Association-LISA Geographical Analysis 27: 93-115

Baker S, Holt KE, Clements ACA, Karkey A, Arjyal A, Boni MF, Dongol S, Hammond N, Koirala S, Duy PT, Nga TVT, Campbell JI, Dolecek C, Basnyat B, Dougan G, Farrar JJ. 2011, Combined highresolution genotyping and geospatial analysis reveals modes of endemic urban typhoid fever transmission, Open Biol 1: 110008.

Ben-Daoud M., Mouhaddach O., Essahlaoui A., Layachi A., Kestemont MP., El Jaafari S., 2011, Conception d'un SIG pour l'évaluation de l'impact des activités anthropiques sur la qualité des eaux superficielles de la ville de Meknès (Maroc), Cahiers de l'ASEES 16 (1), 17-25.

Bos R., Carr R., Keraita B., 2011, Évaluer et atténuer les risques sanitaires associés aux eaux usées dans les pays à faible revenu, In Drechsel P., Scott C., Raschid-Sally L., Redwood M. , Bahri A., L'irrigation avec des eaux usées et la santé: évaluer et atténuer les risques dans les pays à faible revenu, Canada : Presses de l'Université du Québec, pp 31-50.

Carpenter T.E., 2011, The spatial epidemiologic (r)evolution: A look back in time and forward to the future, Spatial and Spatio-temporal Epidemiology 2, 119-124

Charron D., Fleury M., Lindsay L.R., Ogden N, Schuster C. 2008, Répercussions des changements climatiques sur les maladies transmises par l'eau, les aliments, les vecteurs et les rongeurs In. Séguin J., Santé et changements climatique, Ottawa : Santé Canada, P: 195-224.

Cheng Y-J, Tang F-Y, Bao CJ, Zhu Y-F, Liang Q, et al. , 2013, Spatial analyses of typhoid fever in Jiangsu province, People's Republic of China, Geospatial Health 7(2), pp. 279-288.

Chevalier P, Cordier S, Dab W, Gérin M, Gosselin P, et Quénel P, 2003, Santé environnementale. In: Gosselin P, Cordier S, Viau C, Quénel P, Dewailly É, Gérin M, Environnement et santé publique Fondements et pratiques, Paris : Edisem/Tec\&Doc, pp. 59-86. 
Corner R, Dewan AM and Hashizume M., 2013, Modelling typhoid risk in Dhaka Metropolitan Area of Bangladesh: the role of socio-economic and environmental factors. International Journal of Health Geographics, 12:13.

Dewan AM, Corner R, Hashizume M, Ongee ET (2013) Typhoid Fever and Its Association with Environmental Factors in the Dhaka Metropolitan Area of Bangladesh: A Spatial and Time-Series Approach. PLoS Negl Trop Dis 7(1): e1998.

Direction de l'Epidémiologie et de Lutte contre les Maladies (DELM), 2008, Bulletin épidémiologique des maladies sous surveillance, Rabat : Ministère de la santé du royaume du Maroc, $\mathrm{N}^{\circ}$ 65-66-67 et 68.

Direction Provinciale d'Agriculture de Meknès (DPA), 2007, Monographie Agricole de la zone d'action de la Direction Provinciale d'Agriculture de Meknès, Meknès : Direction Provinciale d'Agriculture de Meknès.

Field A., 2009, Discovering statistics using SPSS, Third Edition, London: SAGE Publications.

Gaudart J., Giorgi R., Poudiougou B., Touré O., Ranque S., Doumbo O., Demongeot J., 2007, Détection de clusters spatiaux sans point source prédéfini : utilisation de cinq méthodes et comparaison de leurs résultats, Revue d'épidémiologie et de santé publique, Vol. 55, p 297-306.

Getis A. et Ord J.K., 1992, The Analysis of Spatial Association by Use of Distance Statistics, Geographical Analysis 24, $\mathrm{n}^{\circ} .3$.

Goria S, Stempfelet M, de Crouy-Chanel P., 2011, Introduction aux méthodes statistiques et aux systèmes d'information géographique en santé environnement - Application aux études écologiques - Résultats 2010. Saint-Maurice: Institut de veille sanitaire.

Grégoire M., 2012, Les exploitations du bassin versant de l'Oued Boufekrane à Meknès (Maroc) : situation agro-économique, valorisation des eaux usées et relations avec la ville, Mémoire d'Ingénieur, AgroSup Dijon, Institut national supérieur des sciences agronomiques, de l'alimentation et de l'environnement, soutenue Septembre 2012.

Haut Commissariat au Plan (HCP), 2014, Cartographie des indicateurs communaux de la pauvreté, de la vulnérabilité et de l'inégalité, Adresse URL: http://www.hcp.ma/Indicateurs-communauxde-la-pauvrete-de-la-vulnerabilite-et-de-l-inegalite_a670.html , consulté: Novembre 2014

Haut-Commissariat au Plan (HCP), 2004, Caractéristiques démographiques de la province de Meknès, Recensement Générale de la Population et de l'Habitat, Rabat : Haut-Commissariat au Plan.

Hinman S, Blackburn JK and Curtis A, 2006, Spatial and temporal structure of typhoid outbreaks in Washington, D.C., 1906-1909: evaluating local clustering with the Gi* statistic, International Journal of Health Geographics, 5:13.

Kulldorf M., 1997, A spatial scan statistic. Commun Statist Theory Meth, 26:1481-96.

Kulldorff M, Huang L, Pickle L, Duczmal L., 2006, An elliptic spatial scan statistic, Stat Med 25(22): 3929-43.

Larif M., Soulaymani A., Hnach M., El Midaout A., 2013, Contamination spatio-temporelle d'origine hydrique de l'oued Boufekrane dans la région de Meknès-Tafilalt (Maroc), Int. J. Biol. Chem. Sci. 7(1): 172-184.

Luby S.P., 2014, Bacteria: Salmonella Typhi and Salmonella Paratyphi, Encyclopedia of Food Safety, Volume 1: History, Science and Methods, Pages 515-522. 
Ministère de la Santé du Royaume du Maroc (MS), 2009, Santé en chiffre 2008, Maroc : Direction de la Planification et des Ressources Financières, Division de la Planification et des Etudes Service des Etudes et de l'Information Sanitaire.

Ministère de la Santé du Royaume du Maroc (MS), 2010, Santé en chiffre 2009, Maroc : Direction de la Planification et des Ressources Financières, Division de la Planification et des Etudes Service des Etudes et de l'Information Sanitaire.

Ministère de la Santé du Royaume du Maroc (MS), 2011, Santé en chiffre 2010, Maroc : Direction de la Planification et des Ressources Financières, Division de la Planification et des Etudes Service des Etudes et de l'Information Sanitaire.

Mogasale V., Maskery B., Ochiai R.L., Lee J.S., Mogasale V.V., Ramani E., Kim Y.E., Park J.K., Wierzba T.F., 2014, Burden of typhoid fever in low-income and middle-income countries: a systematic, literature-based update with risk-factor adjustment, The Lancet Global Health, Vol. 2, $\mathrm{N}^{\circ} 10$, e570-e580, October 2014.

Oliveau, S., 2010, Autocorrélation spatiale : leçons du changement d'échelle, L’Espace Géographique, $\mathrm{n}^{\circ}$ 1, pp. 51-64.

Ord J.K. et Getis A., 1995, Local Spatial Autocorrelation Statistics: distributional issues and an application Geographical Analysis, 27(4) 286-306

Organisation Mondiale de la Santé (OMS), 2007, Prévenir la maladie grâce à un environnement sain : une estimation de la charge de morbidité imputable à l'environnement-Résumé, Suisse: Bibliothèque de l'Organisation Mondiale de la Santé.

Organisation Mondiale de la Santé (OMS), 2012, Directives OMS pour l'utilisation sans risque des eaux usées, des excreta et des eaux ménagères, Volume II : Utilisation des eaux usées en agriculture, Genève : Organisation Mondiale de la Santé.

Ouattar, 2010, Réutilisation des eaux usées traitées en agriculture - Projet pilote de Meknès, Rabat : Agence des États-Unis pour le développement international USAID/Maroc.

Pfeiffer D, Robinson T, Stevenson M, Stevens K, Rogers D, Clements C.A., 2008, Spatial Analysis in Epidemiology, New York: Oxford University Press

Polonsky JA, Martı'nez-Pino I, Nackers F, Chonzi P, Manangazira P, et al. , 2014, Descriptive Epidemiology of Typhoid Fever during an Epidemic in Harare, Zimbabwe, 2012. PLoS ONE 9(12):e114702.

RADEEM, 2003, Mission D : Etude environnementale et impact socio-économique, sous mission D3 : Suivi environnementale et impact socio-économique - Recensement des exploitations des perimètres de reutilisation des eaux usées, Rapport du Groupement d'Ingénieurs Conseils, RADEEM, p. 5-12.

Rezaeian M, Dunn G, Leger SS, Appleby L, 2007, Geographical epidemiology, spatial analysis and geographical information systems: a multidisciplinary glossary. J Epidem Comm Health 61:98102.

Rhaidour M. 2013, Durabilité des exploitations maraîchères en zone urbaine et périurbaine de la ville de Meknès (Maroc), Mémoire d'Ingénieur, Ecole Nationale d'Agriculture de Meknes (Maroc), soutenue Octobre 2013.

Sur D, Ali M, Seidlein LV, Manna B, Deen JL, Acosta CJ, Clemens J and Bhattacharya SK, 2007, Comparisons of predictors for typhoid and paratyphoid fever in Kolkata, India, BMC Public Health, 7:289. 
Texier G., Gaudart J., Queyriaux B., 2011, Techniques d'analyse spatiale. In Astagheau P. et Ancelle T., Surveillance épidémiologique: Principes, méthodes et applications en santé publique.

Médecine Sciences Publications: Lavoisier, p. 57-66.

Tran A., Guis H., Guernier V., Gerbier G., 2009, Epidémiologie spatiale : les maladies vues du ciel In. Guégan J-F., Choisy M. Introduction à l'épidémiologie intégrative des maladies infectieuses et parasitaires. Bruxelles : de boeck, p. 41-79.

Waller L.A., Gotway C.A., 2004, Applied Spatial Statistics for Public Health Data, New Jersey: Wiley series in probability and statistics.

Wang J-F, Wang Y, Zhang J, Christakos G, Sun J-L, et al. , 2013, Spatiotemporal Transmission and Determinants of Typhoid and Paratyphoid Fever in Hongta District, Yunnan Province, China.

PLoS Negl Trop Dis 7(3): e2112.

Zitane A.H., 2009, Apport de la télédétection spatiale haute résolution et le SIG dans l'identification, la délimitation et la gestion du périmètre de réutilisation des eaux usées à Meknès au Maroc, Mémoire d'Ingénieur, Institut Agronomique et Vétérinaire Hassan II Rabat (Maroc), soutenue le 10/09/2009.

\section{NOTES}

1. Risque Relatif : c'est l'estimation du risque au sein du cluster divisée par l'estimation du risque en dehors du cluster. $\mathbf{R R}=\left(\mathbf{O}_{\text {int }} / \mathbf{E}_{\text {int }}\right) /\left(\mathbf{O}_{\text {ext }} / \mathbf{E}_{\text {ext }}\right)$ où, $\mathrm{O}_{\text {int }}$ et $\mathrm{E}$ int représentent respectivement les effectifs observées et théoriques dans la fenêtre, et, $\mathrm{O}_{\text {ext }}$ et $\mathrm{E}$ ext représentent les effectifs observées et théoriques à l'extérieur de la fenêtre (Gaudart et al. 2007).

\section{RÉSUMÉS}

La fièvre typhoïde pose un problème majeur de santé publique au niveau de la ville de Meknès (Maroc) avec une incidence annuelle qui dépasse la moyenne nationale. La présente étude vise à analyser la distribution spatio-temporelle de la fièvre typhoïde à l'échelle de la ville durant la période 2008-2010. Les analyses ont permis d'élucider un gradient de l'incidence de la fièvre typhoïde, avec une agrégation des zones à haut risque au nord-ouest de la ville et les zones à bas risque au sud-est. Par approche spatio-temporelle, le dit gradient s'observe durant la période estivale. L'analyse de la corrélation non paramétrique a révélé une association statistiquement significative entre l'incidence de la fièvre typhoïde et la proximité aux périmètres de la réutilisation des eaux usées en agriculture. Aucune relation significative n'a été trouvée avec le taux d'analphabétisme et les indicateurs de la salubrité d'habitat.

Background: With an annual incidence above the national average, typhoid fever is a major public health problem in Meknes city (Morocco). The factors influencing the spatial and temporal distribution of this disease across the city are poorly understood.

Health officers suspect that wastewater is reused to a high extent to irrigate agricultural land in 
urban and suburban areas. However, illiteracy rates, availability of basic amenities and socioeconomic disparities may also influence the spatial distribution of the disease.

Analysis of the geographical distribution of typhoid in Meknes city, as well as the study of its relation to potential risk factors, is important in developing an efficient system for the monitoring and prevention of this food and waterborne disease.

Methods: This study aims to analyse the spatio-temporal pattern of typhoid fever in Meknes city over the period 2008-2010, considering the following hypotheses: (i) the typhoid incidence has a tendency to aggregate in our study area; (ii) the typhoid incidence is higher among the people living close to fields irrigated by wastewater; and (iii) the illiteracy rate and the availability of basic amenities (kitchen, toilet and bath) can influence the disease distribution at local level.

The data concerning the patients with typhoid fever were obtained from the Provincial Delegation of Public Health in Meknes, geocoded to sub-communal tract level and used in spacetime analysis together with demographic, educational, environmental and basic amenities variables.

In addition to addressing the spatio-temporal pattern of typhoid fever, we attempt to provide an overview of the environmental factors: the reuse of wastewater in urban and suburban areas.

Results: Wastewater reuse for agriculture is common in Morocco, but particularly in Meknes city, where it occurs over approximately 2245 ha. The areas where wastewater is reused are distributed over the valleys of three rivers: Bouisshak, Boufekrane and Ouislane.

The Bouisshak area is the most affected by this practice ( $93 \%$ of the area is irrigated by raw wastewater), followed by Ouislane (71\%), and finally Boufekrane (69\%). Vegetables from these areas supply a large quantity of the produce sold at the informal markets of Meknes city; this creates a health risk to consumers. Thus, we investigate the distribution of the health risk associated with typhoid fever in Meknes city. The general spatial autocorrelation analyses have rejected the null hypothesis corresponding to a random distribution during the years 2008 $(p<0.05)$ and $2010 \quad(p<0.01)$. These results show that the typhoid fever incidence tends to aggregate in Meknes city.

To confirm this finding and to understand this trend, we proceeded with the mapping of the high and low risk clusters, using local spatial autocorrelation analyses. The spatial scan of Kulldorff has elucidated the existence of a spatial gradient. Specifically, the high risk clusters were mainly aggregated in the Northwest of the city, close to the areas of wastewater reuse, whereas the low risk clusters in the Southeast.

According to the spatio-temporal approach, this gradient occurs during the summer season (May-October) due to the intensive use of wastewater in agriculture and scarcity of water resources during this period.

Nonparametric correlation has revealed a statistically significant association between the incidence of typhoid fever and the proximity to areas of wastewater reuse in agriculture. No statistical association was found with the illiteracy rate and basic amenities variables.

Conclusion: A better understanding of the spatial distribution patterns of typhoid fever will help to identify areas and populations at high risk and assist public health officers of Meknes city to plan actions to reduce the risk of food and waterborne disease.

\section{INDEX}

Mots-clés : fièvre typhoïde, distribution spatio-temporelle, analyse statistique Index géographique : Meknes, Maroc 
AUTEUR

OMAR MOUHADDACH

Université Moulay Ismail - Faculté des Sciences de Meknès (Maroc) 